\title{
Static Dipole Moments and Electronic Structure Calculations of the Low-Lying Electronic States of the Molecule Zinc Selinum ZnSe
}

\author{
Abeer Youssef $^{1}$, Ghassan Younes ${ }^{1} \&$ Mahmoud Korek ${ }^{1}$ \\ ${ }^{1}$ Faculty of Science, Beirut Arab University, Lebanon \\ Correspondence: Mahmoud Korek, Faculty of science, Beirut Arab University, P.O.Box 11-5020, Beirut, \\ Lebanon. E-mail: mahmoud.korek@bau.edu.lb, fkorek@yahoo.com
}

Received: May 1, 2017

doi:10.5539/mas.v11n9p14

\author{
Accepted: June 11, 2017 \\ Online Published: August 4, 2017 \\ URL: https://doi.org/10.5539/mas.v11n9p14
}

\begin{abstract}
Zinc selenide is a compound that has many applications in optoelectrical systems. An understanding of its properties as an individual molecule can be of great help for its use at the nanoscale. Correspondingly, twenty two lowest electronic states of $\mathrm{ZnSe}$ have been studied in the ${ }^{2 \mathrm{~s}+1} \Lambda^{ \pm}$representation in this paper. The potential energy curves, the harmonic frequency $\omega_{e}$, the electronic energy $T_{e}$, the static dipole moment and the internuclear distance $r_{e}$ have been investigated. These calculations have been performed by using the multi-reference configuration interaction (MRCI+Q) method with Davidson correction. A very good agreement is obtained by comparing the present results with those available in literature. New electronic states have studied in the present work for the first time.
\end{abstract}

Keywords: ab initio calculation, electronic structure, spectroscopic constants, potential energy curves

\section{Introduction}

Zinc is one of the essential elements for humans by its effects as cofactor for a very large number of enzymes, zinc-finger proteins and matrix metalloproteinases. The calculations of the dipole moment and the bond dissociation of $\mathrm{Zn}$ compounds provide critical data for biological simulations and industrial applications (Auld 2001), and (Silva, \& Williams 2001). Zinc Chalcogenides have large iconicity of chemical bond with small values of energy for the formation of vacancies. From a stoichiometric ratio of $\mathrm{Zn}$ and Se powder, $\mathrm{ZnSe}$ molecule can be obtained by microwave irradiation technique $(2.8 \mathrm{GHz})$. These compounds are very promising in optoelectronic applications, i.e in the domains of infrared optics and electro-optic, lenses, laser diodes and electric diodes, beam expanders, semiconductors, and solar cells (Wu, Qiu, Cai, Xu, Chen, \& Cryst 2002), and (Porento, \& Hirva 2002). The study of the structure and the electronic properties of these compounds at a small scale are needed to understand the applications of these materials. For example, ZnSe nanocluster represents the link between molecules and the bulk of these materials. At the molecular scale, theoretical investigations of the electronic structure of $\mathrm{ZnSe}$ are valuable in order to understand their experimentally observed properties. However, no systematic theoretical investigation on the potential energy, the static dipole moment, and the spectroscopic constants of $\mathrm{ZnSe}$ molecules have been conducted thus far. In the present work and in order to get further insight into various properties of $\mathrm{ZnSe}$ molecule, we extend our investigation to their highly excited electronic states with a rovibrational calculation using the canonical function approach.

\section{Method}

By using an ab initio calculation, we investigate in the present work the low-lying electronic states of $\mathrm{ZnSe}$ molecule. The calculation has been performed via CASSCF method. In order to determine the correlation effect, multireference CI calculations were performed using Davidson correction with singly and doubly excitations. In the MRCI calculation, all the CASSCF configuration space has been used as a reference. These calculations have been performed by using the computational chemistry program MOLPRO (MOLPRO 2015) with the graphical user gabedit interface (Allouche 2011). The $\mathrm{Zn}$ atom is treated by using the ECP10MDF basis set for the s, $\mathrm{p}$, and d orbitals while the ECP28MWB basis is used for Se atom for the $s$ and $p$ orbitals; the $d$ orbital for this atom is added from the aug-cc-PVDZ;C basis. Among the 24 electrons explicitly considered for the molecule $\mathrm{ZnSe}, 4$ electrons were frozen in our calculation so that 9 valence electrons were explicitly treated. Around the equilibrium position, the molecule $\mathrm{ZnSe}$ can be considered ionic as many other transition metals. 


\section{Results}

\subsection{Potential Energy Curves and Spectroscopic Constants}

In the representation ${ }^{2 s+1} \Lambda^{(+/)}$, the calculation of the eleven singlet and eleven triplet potential energy curves (PECs) of the ZnSe molecule has been performed up to 96 internuclear distances in the range of $1.49 \AA<\mathrm{r}<$ 4.5 $\AA$. These curves for the electronic states ${ }^{1,3} \Sigma^{ \pm},{ }^{1,3} \Pi$, and ${ }^{1,3} \Delta$ in the considered range of $\mathrm{R}$ are given respectively in Figures 1-5. One can notice that some avoided crossings have been obtained between the potential energy curves $(2)^{1} \Sigma^{+} /(3)^{1} \Sigma^{+},(1)^{1} \Pi /(2)^{1} \Pi,(2)^{1} \Pi /(3)^{1} \Pi,(3)^{1} \Pi /(4)^{1} \Pi,(2)^{3} \Pi /(3)^{3} \Pi$ and $(4)^{3} \Pi /(5)^{3} \Pi$. At these avoided crossing the corresponding wave functions will mix with each other to give two adiabatic solutions. These solutions of the Schrödinger equation are obtained by linear combinations of the diabatic ones where the variation method is used. Such crossings or avoided crossings can dramatically alter the stability of the molecule. Because crossing or avoided crossing is near the minima of some of the investigating potential energy curves, the spectroscopic constants for these curves have not been calculated.

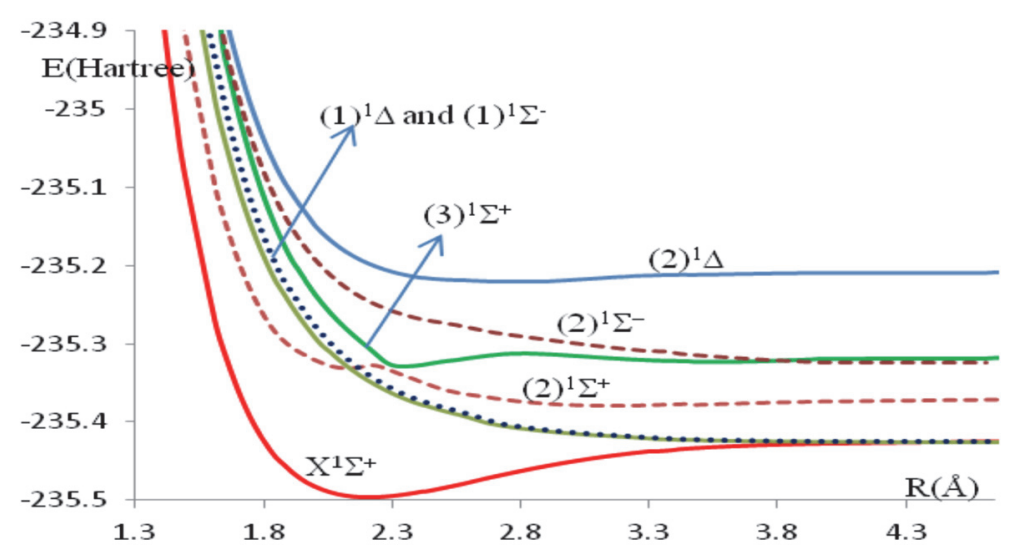

Figure 1. Potential Energy Curves of the Singlet ${ }^{1} \Sigma^{ \pm}$and ${ }^{1} \Delta$ Electronic States of the Molecule ZnSe

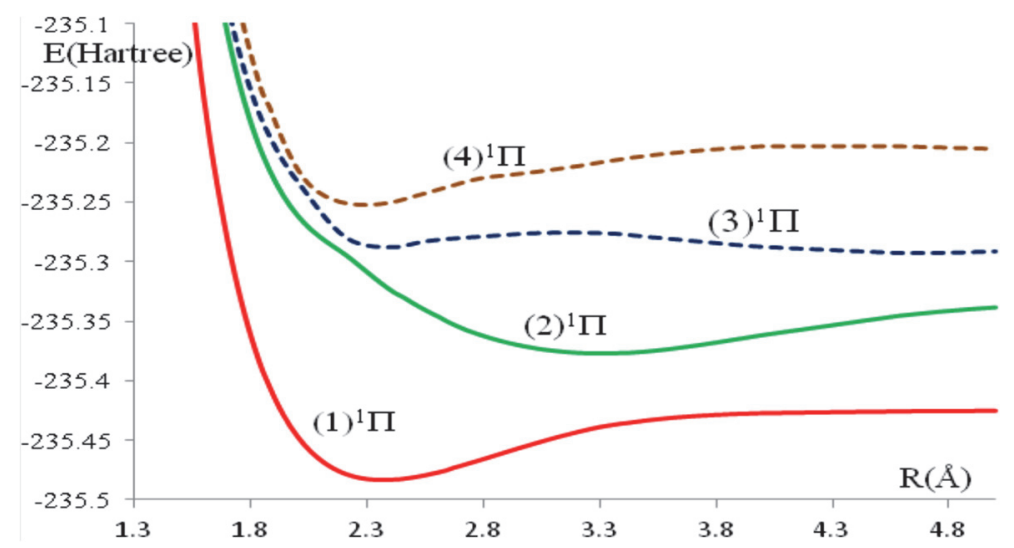

Figure 2. Potential Energy Curves of the Singlet ${ }^{1} \Pi$ Electronic States of the Molecule ZnSe.

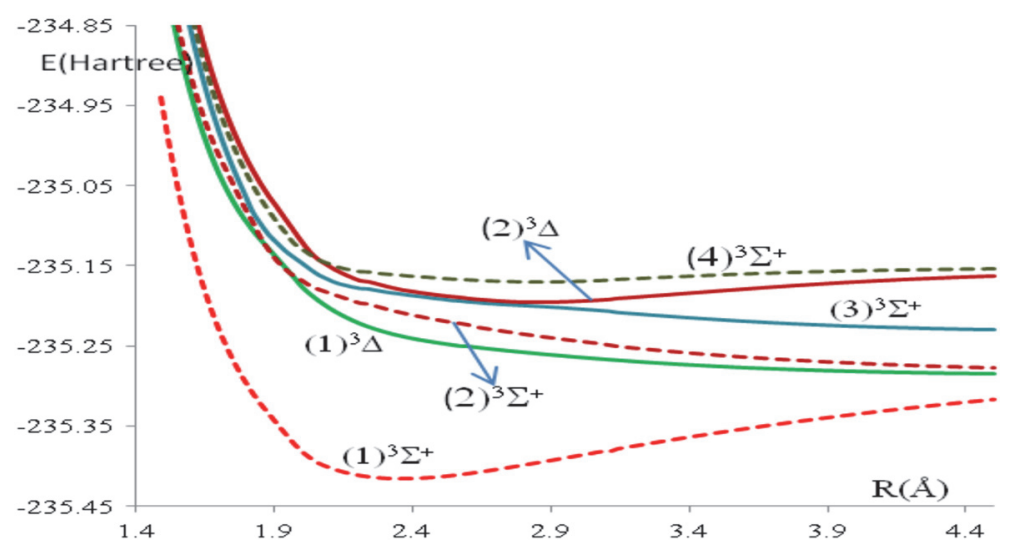

Figure 3. Potential Energy Curves of the Triplet ${ }^{3} \Sigma^{+}$and ${ }^{3} \Delta$ Electronic States of the Molecule ZnSe 


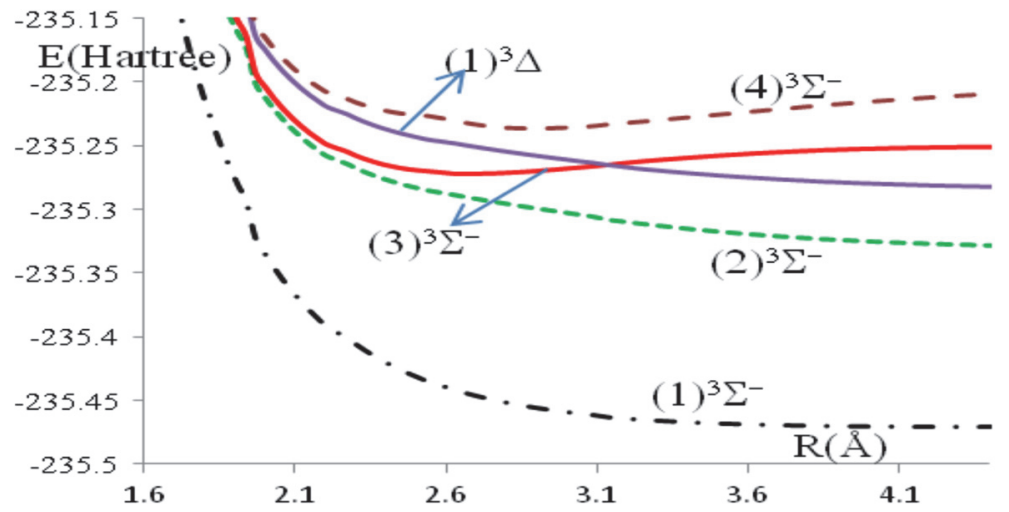

Figure 4. Potential Energy Curves of the Triplet ${ }^{3} \Sigma^{-}$and ${ }^{3} \Delta$ Electronic States of the Molecule $\mathrm{ZnSe}$

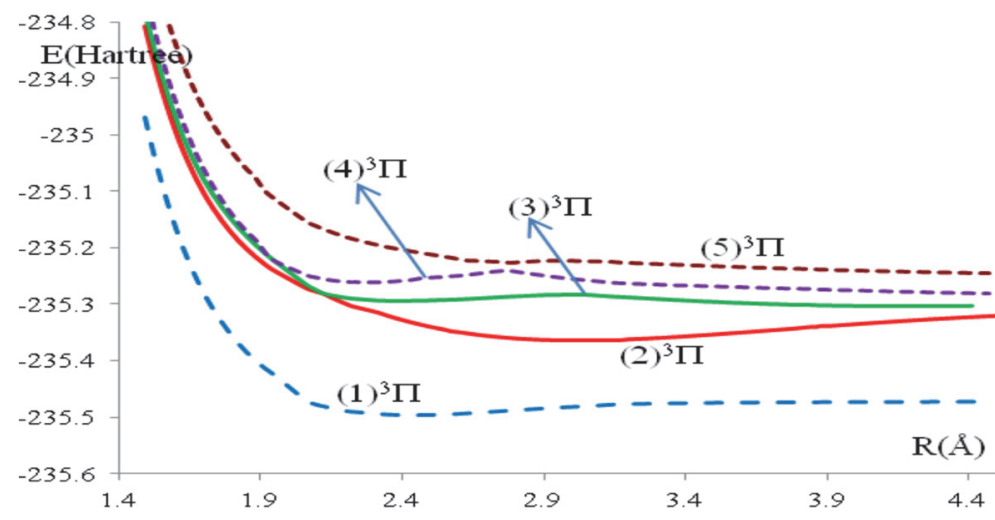

Figure 5. Potential Energy Curves of the Triplet ${ }^{3} \Pi$ Electronic States of the Molecule ZnSe

For each investigated electronic state, the relative energy with respect to the ground state $T_{e}$, the harmonic frequencies $\omega_{\mathrm{e}}$, the rotational constants $\mathrm{B} e$, and the internuclear distance at equilibrium $\mathrm{Re}_{\mathrm{e}}$ for the singlet and triplet electronic states of the $\mathrm{ZnSe}$ molecule have been calculated by fitting the calculated energy values of the different investigated electronic states into a polynomial in $\mathrm{R}$ around the equilibrium values $\mathrm{Re}$. These values along with those found in literature, either theoretical or experimental, are given in Table 1.

Table 1. Spectroscopic Constants of the Zinc Selinum Molecule ZnSe

\begin{tabular}{lllcc}
\hline States & \multicolumn{1}{c}{$\mathrm{T}_{\mathrm{e}}\left(\mathrm{cm}^{-1}\right)$} & $\omega_{\mathrm{e}}\left(\mathrm{cm}^{-1}\right)$ & $\mathrm{B}_{\mathrm{e}}\left(\mathrm{cm}^{-1}\right)$ & $\mathrm{R}_{\mathrm{e}}(\AA)$ \\
\cline { 3 - 5 } $\mathrm{X}^{1} \Sigma^{+}$ & $0.0^{\mathrm{a}}$ & 298.6 & 0.0965 & 2.2085 \\
& $0.0^{\mathrm{b} 1}$ & 328.9 & & 2.1752 \\
\cline { 3 - 5 } & $0.0^{\mathrm{b} 2}$ & 238.5 & 2.1738 \\
\hline & $0.0^{\mathrm{b3}}$ & & & 2.163 \\
\hline$(1)^{1} \Pi$ & $2900.04^{\mathrm{a}}$ & 252.1 & 0.084 & 4.475 \\
\hline$(1)^{3} \Pi$ & $3536.2^{\mathrm{a}}$ & 398.88 & 0.283 & 2.436 \\
\hline & $3315.7^{\mathrm{b} 3}$ & 238.5 & & 2.353 \\
\hline$(1)^{3} \Sigma^{-}$ & $17674.8^{\mathrm{a}}$ & 799.94 & 0.286 & 4.577 \\
$(2)^{1} \Sigma^{+}$ & $25726.5^{\mathrm{a}}$ & - & 0.051 & 5.748 \\
$(2)^{1} \Pi$ & $26103.4^{\mathrm{a}}$ & 136.2 & 0.043 & 6.254 \\
$(2)^{3} \Pi$ & $28875.2^{\mathrm{a}}$ & 277.61 & 0.181 & 5.745 \\
$(3)^{1} \Pi$ & $45666.0^{\mathrm{a}}$ & 306.73 & 0.083 & 5.058 \\
$(3)^{3} \Sigma^{-}$ & $49221.0^{\mathrm{a}}$ & 322.6 & 0.234 & 4.293 \\
$(4)^{3} \Pi$ & $51782.5^{\mathrm{a}}$ & 322.6 & 0.091 & 4.293 \\
$(4)^{1} \Pi$ & $53555.8^{\mathrm{a}}$ & 286.5 & 0.089 & 4.330 \\
\hline$(2)^{1} \Sigma^{-}$ & $58147.7^{\mathrm{a}}$ & 383.1 & 0.055 & 5.520 \\
\hline
\end{tabular}


${ }^{\text {a Present Work, }}{ }^{\mathrm{b} 1}($ Peterson, Shepler, \& Singleton-CCSD $(\mathrm{T})),{ }^{\mathrm{b} 2}$ (Peterson, Shepler,

\& Singleton -MRCI+Q), ${ }^{\text {b3 }}$ (Peterson, Shepler, \& Singleton - using frozen-core MRCI+Q/CBS)

The comparison of our calculated value of the internuclear distance Re with those given in literature for the ground state (Peterson, Shepler, \& Singleton 2007) showed a very good agreement with the average relative difference $\Delta \mathrm{Re}_{\mathrm{e}} / \mathrm{Re}_{\mathrm{e}}=1.5 \%$; this relative difference becomes $3.4 \%$ for the first excited electronic state $(1)^{3} \Pi$. In literature, there are only 2 theoretical published values for the harmonic vibrational frequency $\omega_{\mathrm{e}}$ for the ground state where the difference between them is $90.4 \mathrm{~cm}^{-1}$ (Peterson, Shepler, \& Singleton 2007). Our calculated value for this constant is closer to that given by (Peterson, Shepler, \& Singleton-CCSD(T) 2007), with the relative difference $\Delta \omega_{\mathrm{e}} / \omega_{\mathrm{e}}=9.1 \%$ while it is in disagreement with the value given by (Peterson, Shepler, \& Singleton -MRCI+Q 2007), where the relative difference is $25.2 \%$. Our calculated value of these constants for the first excited state is smaller than that given by (Peterson, Shepler, \& Singleton 2007). There is no comparison of our calculated values of the spectroscopic constants with other data in literature since they are given here for the first time.

\subsection{Static Dipole Moment}

The vibrational excitation of a molecule depends on the variation of the dipole moment in terms of the internuclear distance. These static dipole moment curves can help in the transition intensities. By taking the atom $\mathrm{Zn}$ at the origin, we plot in Figures 6-7 the static dipole moment curves versus the internuclear $\mathrm{R}$ for singlet and triplet electronic states of the molecule $\mathrm{ZnSe}$.

$\mu($ a.u. $)$

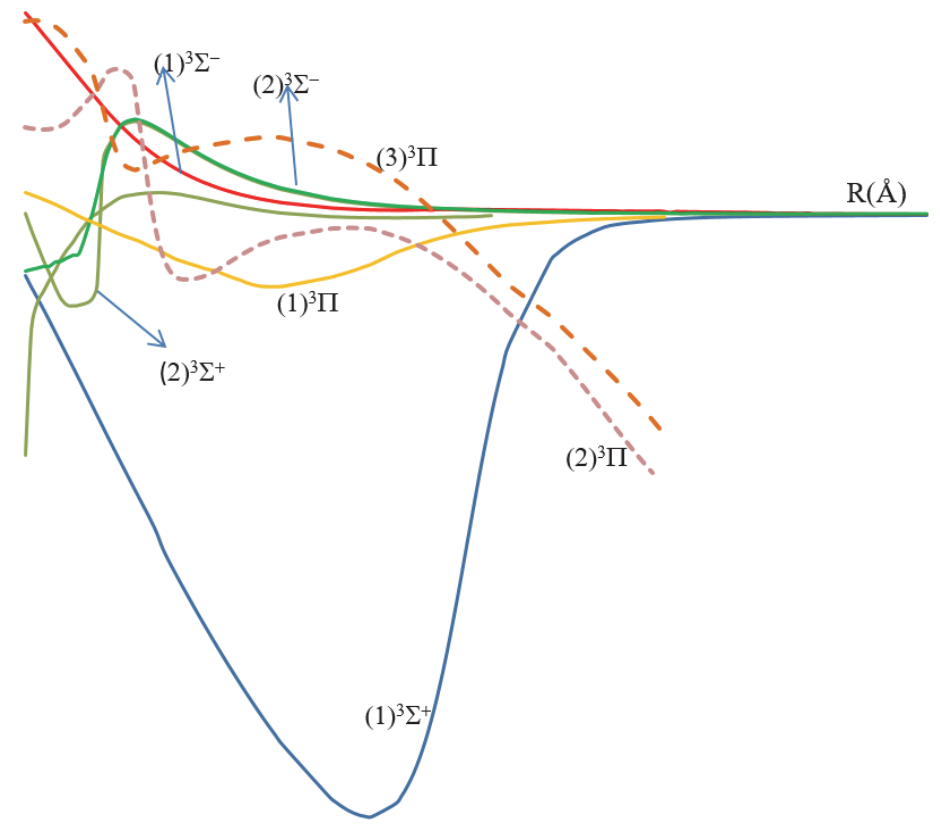

Figure 6. Static Dipole Moment Curves of Triplet Electronic States of the Molecule ZnSe.

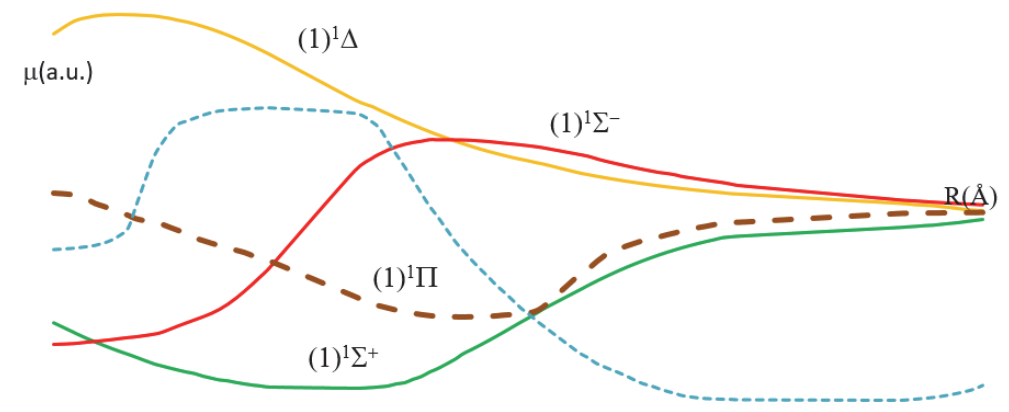

Figure 7. Static Dipole Moment Curves of Singlet Electronic States of the Molecule ZnSe 
At large value of the internuclear distances $\mathrm{R}$, the dipole moment curves of the molecule $\mathrm{ZnSe}$ tend to approach constant value or zero smoothly where the molecule dissociates into its natural fragments. The advancement in the study of cold and ultracold molecules offers new techniques for precision tests of fundamental physics theories. The vibrational intervals in diatomic molecules are of interest in the context of such an enhancement. Within the Born-Oppenheimer approximation, the radial Schrödinger equation can be replaced, by using the canonical functions approach (Kobeissi, Korek, \& Dagher 1989), and (Korek 1999) where the eigenvalues Ev, the rotational constants $\mathrm{B}_{\mathrm{v}}$, and the centrifugal distortion constants $\mathrm{D}_{\mathrm{v}}$ have been calculated for the electronic state $(1)^{1} \Sigma^{+}$. These values are given in Table 2 .

Table 2. The Eigenvalue Ev, the Rotational Constant Bv and the Centrifugal Distortion Constant Dv of the Molecule $\mathrm{ZnSe}$

\begin{tabular}{|c|c|c|c|}
\hline $\mathrm{V}$ & $\mathrm{E}_{\mathrm{v}}\left(\mathrm{cm}^{-1}\right)$ & $\mathrm{B}_{\mathrm{v}} \times 10^{2}\left(\mathrm{~cm}^{-1}\right)$ & $D_{v} \times 10^{2}\left(\mathrm{~cm}^{-1}\right)$ \\
\hline 0 & 134.9 & 9.415 & 4.897 \\
\hline 1 & 394.8 & 9.354 & 4.305 \\
\hline 2 & 661.5 & 9.344 & 4.573 \\
\hline 3 & 926.7 & 9.263 & 4.915 \\
\hline 4 & 1187.8 & 9.254 & 4.091 \\
\hline 5 & 1450.6 & 9.177 & 5.075 \\
\hline 6 & 1709.3 & 9.172 & 3.654 \\
\hline 7 & 1971.1 & 9.123 & 3.958 \\
\hline 8 & 2234.1 & 9.140 & 3.229 \\
\hline 9 & 2500.8 & 9.095 & 4.862 \\
\hline 10 & 2763.6 & 9.032 & 6.475 \\
\hline 11 & 3017.6 & 8.939 & 4.461 \\
\hline 12 & 3269.7 & 8.930 & 3.677 \\
\hline 13 & 3523.0 & 8.849 & 4.946 \\
\hline 14 & 3773.1 & 8.823 & 5.433 \\
\hline 15 & 4019.5 & 8.791 & 3.839 \\
\hline 16 & 4265.7 & 8.746 & 5.028 \\
\hline 17 & 4509.2 & 8.695 & 4.954 \\
\hline 18 & 4750.3 & 8.654 & 4.364 \\
\hline 19 & 4990.0 & 8.605 & 5.089 \\
\hline 20 & 5227.2 & 8.561 & 4.743 \\
\hline 21 & 5462.4 & 8.512 & 4.790 \\
\hline 22 & 5695.5 & 8.465 & 5.019 \\
\hline 23 & 5926.4 & 8.419 & 4.868 \\
\hline 24 & 6155.1 & 8.368 & 4.896 \\
\hline 25 & 6381.8 & 8.321 & 5.125 \\
\hline 26 & 6606.2 & 8.272 & 4.968 \\
\hline 27 & 6828.4 & 8.221 & 5.063 \\
\hline 28 & 7048.3 & 8.172 & 5.256 \\
\hline 29 & 7265.9 & 8.122 & 5.150 \\
\hline 30 & 7481.2 & 8.069 & 5.233 \\
\hline 31 & 7694.2 & 8.018 & 5.464 \\
\hline 32 & 7904.8 & 7.966 & 5.409 \\
\hline 33 & 8112.9 & 7.911 & 5.439 \\
\hline 34 & 8318.6 & 7.858 & 5.635 \\
\hline 35 & 8521.9 & 7.806 & 5.580 \\
\hline 36 & 8722.7 & 7.754 & 5.409 \\
\hline 37 & 8921.4 & 7.701 & 5.651 \\
\hline 38 & 9117.6 & 7.645 & 6.348 \\
\hline 39 & 9310.9 & 7.577 & 7.027 \\
\hline 40 & 9500.3 & 7.502 & 6.931 \\
\hline
\end{tabular}




\begin{tabular}{c|c|c|c}
\hline 41 & 9686.2 & 7.434 & 6.596 \\
\hline 42 & 9869.0 & 7.361 & 8.511 \\
\hline 43 & 10046.7 & 7.245 & 11.62 \\
\hline 44 & 10217.1 & 7.112 & 10.39 \\
\hline 45 & 10381.4 & 7.020 & 8.005 \\
\hline 46 & 10541.8 & 6.930 & 10.35 \\
\hline 47 & 10696.8 & 6.814 & 10.54 \\
\hline 48 & 10846.9 & 6.741 & 4.158 \\
\hline 49 & 10996.4 & 6.723 & 5.630 \\
\hline 50 & 11144.0 & 6.599 & 17.62 \\
\hline
\end{tabular}

\section{Conclusion}

We have presented in this work a precise description of the electronic states of the molecule ZnSe. The data presented can be of great use for scientists and engineers who are interested to work with the compound at the molecular or nanoscale, for both the ground and excited states. Furthermore, based on this study, spin orbital, Enistein coefficient, transitional dipole moments, and Franck-Condon Factor can be performed as future study.

\section{References}

Allouche, A., \& Gabedit, R. (2011). A Graphical User Interface for Computational Chemistry Software, Journal of Computational Chemestry, 32, 174-182, https://doi.org/10.1002/jcc.21600

Auld D. S, Bertini, I., Sigel, A., \& Sigel, H., (2001). In Handbook on Metalloproteins Eds.; Dekker: New York, p881. https://doi.org/10.1007/978-94-017-8742-0

De Silva, J. J. R. F., \& Williams R. J. P. (2001). The Biological Chemistry of the Elements: The Inorganic Chemistry of Life, $2^{\text {nd }}$ ed.; Oxford University Press: New York, 2001. https://doi.org/10.1021/ed069pA326.1

Kobeissi. H, Korek. M, \& Dagher. M, (1989). On the Computation of Diatomic Centrifugal Distortion Constants: Exact Solution for Initial Value Problems, Journal Molecular Spectroscopy, 38, 1-4. https://doi.org/10.1016/0022-2852(89)90092-1U

Korek. M. (1999). Highly Accurate Diatomic Centrifugal Distortion Constants, Computer Physics Communication, 119, 69. https://doi.org/10.1002/jcc.540130909

MOLPRO is a package of ab initio programs written by H. J. Werner and P. J. Knowles, with contributions from R.D. Amos, A. Bernhardsson, A. Berning, P. Celani, D.L. Cooper, M. J. O. Deegan, A. J. Dobbyn, F. Eckert, C. Hampel, G. Hetzer, T. Korona, R. Lindh, A.W. Lloyd, S.J. McNicholas, F.R. Manby, W. Meyer, M.E. Mura, A. Nicklass, P. Palmieri, R. Pitzer, G. Rauhut, M. Schütz, H. Stoll, A.J. Stone, R. Tarroni and T. Thorsteinsson. Retrieved from https://www.molpro.net/info/2015.1/doc/update/node1.html

Peterson, K. A., Shepler B. C., \& Singleton, J. M. (2007). The Group 12 Metal Chalcogenide: An accurate Multireference Interaction and Couped Cluster Study, Molecular Physics, 105(9), 1139-1155. https://doi.org/10.1080/00268970701241664

Porento, M., \& Hirva, P. (2002). Theoretical Studies on the Interaction of Aniomic Collectrors with $\mathrm{Cu}+, \mathrm{Cu} 2+$, $\mathrm{zn} 2+$ and $\mathrm{Pb} 2+$ ion, Theoretical Chemistry Account, 107, 200-205. https://doi.org/10.1007/s00214-001-0316-8

Wu, H. Z., Qiu, D. J., Cai, Y. J., Xu, X. L., Chen, W. B., \& Cryst. J. G. (2005) Self-assembled zinc oxide nanodots on silicon oxide, Journal of Physics: Conference Series, 10, 121-124. https://doi.org/10.1088/1742-6596/10/1/030

\section{Copyrights}

Copyright for this article is retained by the author(s), with first publication rights granted to the journal.

This is an open-access article distributed under the terms and conditions of the Creative Commons Attribution license (http://creativecommons.org/licenses/by/4.0/). 